\title{
On Timing Properties of LYSO-based Calorimeters
}

\author{
D. Anderson ${ }^{1}$, A. Apresyan ${ }^{1}$, A. Bornheim ${ }^{1}$, J. Duarte ${ }^{1}$, \\ C. Pena ${ }^{1}$, A. Ronzhin ${ }^{2}$, M. Spiropulu ${ }^{1}$, J. Trevor ${ }^{1}$, and S. Xie ${ }^{1}$ \\ ${ }^{1}$ California Institute of Technology, Pasadena, CA, USA \\ ${ }^{2}$ Fermi National Accelerator Laboratory, Batavia, IL, USA
}

\begin{abstract}
We present Test Beam studies and results on the timing performance and characterization of the time resolution of LutetiumYttrium Orthosilicate (LYSO)-based calorimeters. We demonstrate that a time resolution of $30 \mathrm{ps}$ is achievable for a particular design. Furthermore, we discuss precision timing calorimetry as a tool for the mitigation of physics object performance degradation effects due to the large number of simultaneous interactions in the high luminosity environment foreseen at the Large Hadron Collider.
\end{abstract}

\section{Introduction}

The high luminosity upgrade of the Large Hadron Collider (HL-LHC) at CERN [1] is expected to provide instantaneous luminosities of $5 \times 10^{34} \mathrm{~cm}^{-2} \mathrm{~s}^{-1}$. The enhanced data rates will provide the datasets necessary to perform precision measurements of the Higgs couplings, probe rare Higgs processes, study the scattering of longitudinally polarized $\mathrm{W}$ bosons, and search for physics beyond the standard model.

The rate of simultaneous interactions per bunch crossing (pileup) is projected to reach an average of 140 to 200. The large amount of pileup increases the likelihood of confusion in the reconstruction of the events of interest because of the contamination from particles 
produced in different pileup interactions. The ability to discriminate between jets produced in the events of interest - especially those associated with the vector boson fusion processes - and jets produced by pileup interactions will be degraded, the missing transverse energy resolution will deteriorate, and several other physics objects performance metrics will suffer.

One way to mitigate the pileup confusion effects, complementary to precision tracking methods, is to perform a time of arrival measurement associated with a particular layer of the calorimeter, allowing for a time assignment for both charged particles and photons. Such a measurement with a precision of about 20 to $30 \mathrm{ps}$, when unambiguously associated to the corresponding energy measurement, will significantly reduce the inclusion of pileup particles in the reconstruction of the event of interest, given that the spread in collision time of the pileup interactions is approximately $200 \mathrm{ps}$. The association of the time measurement with the energy measurement is crucial, and leads to a prototype design that calls for the time and energy measurements to be performed in the same active detector element. It is in this context that we studied the possibility of measuring the time of arrival of the particles with a calorimetric device.

We focused our studies on the measurements of the time of flight using sampling calorimeters based on LYSO crystals. Due to its very high light yield $(\sim 30 \mathrm{~K}$ photons $/ \mathrm{MeV})$ [2], and radiation tolerance [36], LYSO is the active element of one of the options considered for the upgrade of the Compact Muon Solenoid (CMS) detector for the HL-LHC [7].

Figure 1 shows a simplified illustration of the major time scales associated with the timing measurement using a monolithic crystal calorimeter. Upon entering the crystal, the photon or electron travels at the speed of light, interacts, and begins to shower, producing scintillation light in the crystal. The time between the entry of the photon into the crystal and the first interaction is denoted by $t_{I}$, and, for high energy impinging particles, corresponds to the shower development time. The time associated with the conversion of the incident photon into scintillation light is denoted by $t_{S}$. The scintillation light travels from the point of the interaction to the photodetector at the velocity $c / \hat{n}$, where $\hat{n}$ is the effective index of refraction of the crystal [8]. The time associated with the propagation of the scintillation light to the photodetector is denoted by $t_{P}$. Once the scintillation light reaches the photodetector, the photons are converted into an 
Figure 1: Timing measurement schematic breakdown using a monolithic, large scintillating crystal. The incident particle impinges on the crystal face from the left. The characteristic time intervals are discussed in the text.

electrical signal. The time associated with this process is known as the photodetector signal transit time, $t_{T}$. Finally, the data acquisition (DAQ) system has a characteristic time constant $t_{D}$. Each of these time intervals will fluctuate or jitter on an event-by-event basis, contributing to the time resolution.

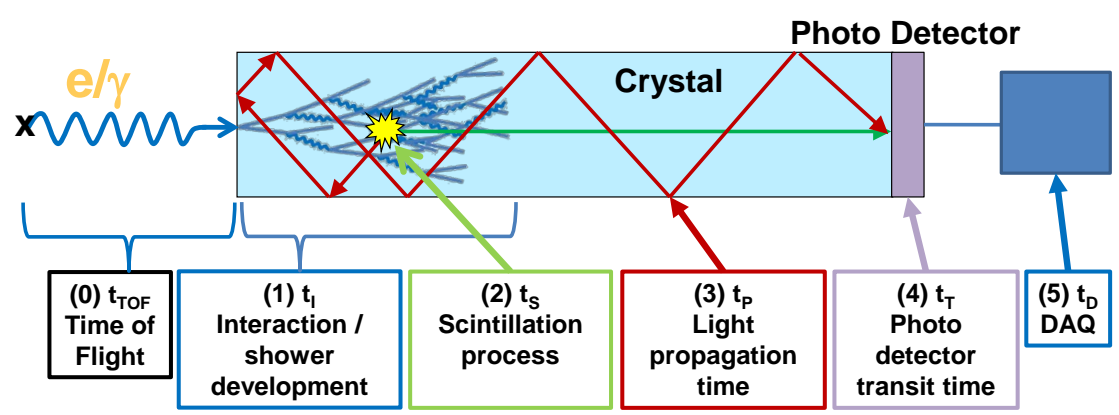


Figure 2: Basic schematic diagram of the experimental setup for a typical timeof-flight measurement shown to illustrate the basic detector elements. One photodetector is used as a time reference, whereas the second measures energy and time simultaneously.

A schematic diagram of a typical time-of-flight measurement setup is shown in Figure 2. All measurements involve a fast photodetector, typically an MCP-PMT, which measures the reference $\left(t_{0}\right)$ timestamp, and a photodetector further downstream, which detects the signal associated with the electromagnetic shower and provides simultaneous energy and time $\left(t_{1}\right)$ measurements.

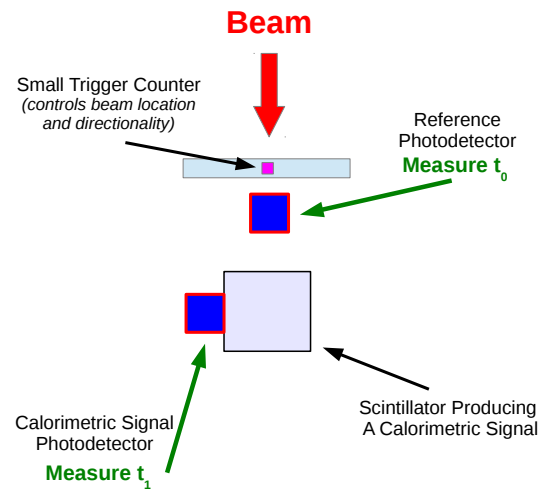

In our study, we used two types of MCP-PMT photodetectors, one
produced by Hamamatsu ${ }^{1}$ (model R3809-52) [10], and one produced
by Photek (model PMT240) [11. [A5] A DRS4 waveform digitizer V4
evaluation board [12] was used as the primary DAQ system, con-
nected to a laptop via USB[A6] interface. The DRS chip contains
a switched capacitor array (SCA) with 1024 cells capable of digitiz-
ing eight analog signals with high speed (5 GSPS) and high accuracy
(11.5 bit SNR). All the experimental beam studies were performed
at the Fermilab Test Beam Facility (FTBF), which provided proton
beams from the Fermilab Main Injector accelerator at $120 \mathrm{GeV}$, and
secondary electron beams of energies ranging from 4 to $32 \mathrm{GeV}$. All

\section{Experimental Setup}

\footnotetext{
${ }^{1}$ Hamamatsu, 250 Wood Ave. Middlesex, NJ 08846 US
} 
the detector elements were placed inside a dark box lined with copper foil to provide radiofrequency shielding. A $2 \times 2 \mathrm{~mm}^{2}$ scintillator was placed inside the box at the upstream extremity and used to trigger the DAQ readout, providing a strict constraint on the location and directionality of the beam particles used in the time-of-flight studies. A differential Cherenkov counter (not shown in the schematic), provided by the FTBF and located upstream of our experimental hall, was used for the electron identification.

\section{Event Selection and Data Analysis}

Our primary target was to reconstruct the time of flight of beam particles between different detector elements. Different time reconstruction algorithms are used for different detector elements, and all involve the assignment of a timestamp using specific features of each corresponding signal pulse. The signal pulse for the reference time detector is very sharp and symmetric around its maximum amplitude, as shown in Figure 3. Hence, for the reference detector, we determined the time position of the pulse peak by fitting a Gaussian function to the peak of the pulse, using three sampling points before the pulse maximum and four sampling points after. The fitted mean parameter of the Gaussian function was assigned as the timestamp $t_{0}$. The signal pulse for the downstream time measurement is the result of the scintillation light, and exhibits a fast rising edge and a significantly slower decay. Therefore, we assigned the timestamp $t_{1}$ using a constant fraction of the rising edge. A linear function was fitted to the sampling points between $10 \%$ and $60 \%$ of the pulse maximum, and the timestamp was assigned as the time at which the fitted linear function rises to $20 \%$ of the pulse maximum. Examples of fits performed to assign a timestamp from each pulse are shown in Figure 4. The impact of the choice of the functional forms was studied using a set of alternative functions in the fits, and choosing the one that resulted in the best time resolution. Among the functions that we tested, the difference between the best and worst performing functions was about 8 ps.

Event selection and pulse cleaning procedures are used to eliminate abnormal pulses in the readout, as described in [9]. Large signals above $500 \mathrm{mV}$ were rejected because they saturate the DRS4 inputs. To reduce the impact of the noise originated from the DRS waveform digitizer DAQ system, only pulses with an amplitude larger than 20 

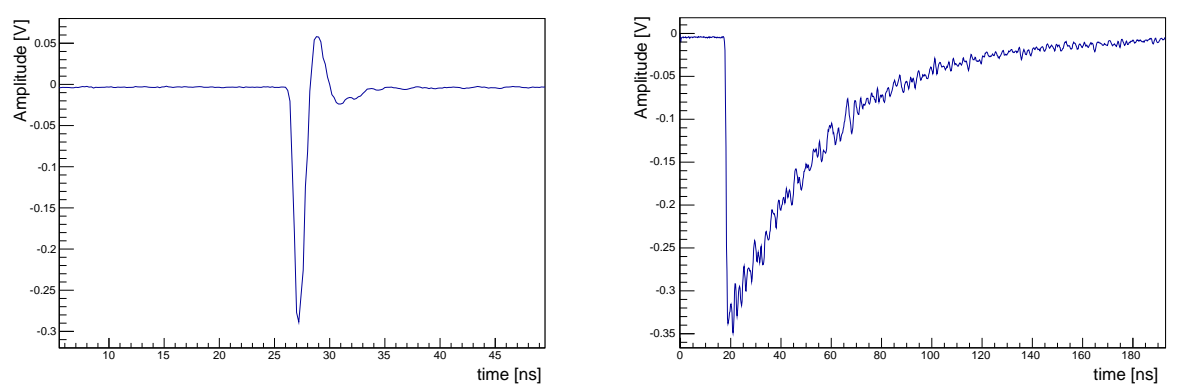

Figure 3: Sample pulses as digitized by the DRS4 board: (left) a pulse from the reference Hamamatsu R3809 MCP-PMT, and (right) a pulse from the Hamamatsu R3809 MCP-PMT optically coupled to a $(1.7 \mathrm{~cm})^{3}$ LYSO crystal cube recorded using an $8 \mathrm{GeV}$ electron beam.

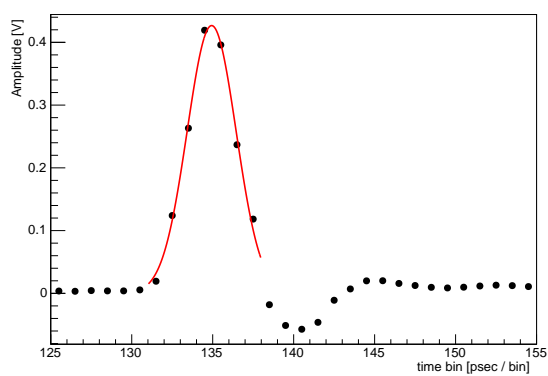
pulses were saturated.
$\mathrm{mV}$ were used for the time-of-flight measurements. Events containing more than one pulse within the 200 ns readout window were not considered. Attenuators were used to extend the dynamic range of the DRS4 waveform digitizer in cases when a large fraction of the signal

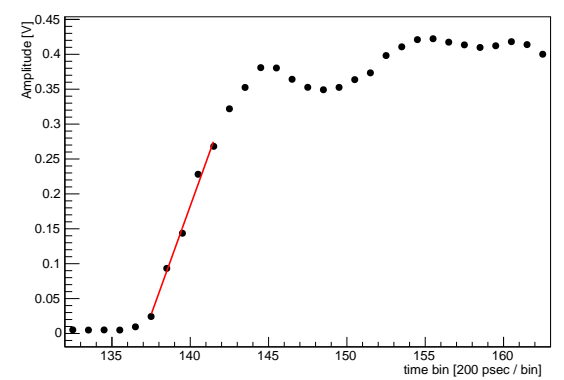

Figure 4: Sample fits used to assign timestamps to digitized MCP-PMT pulses: (left) a pulse from the reference Hamamatsu R3809 MCP-PMT; (right) a pulse from the Hamamatsu R3809 MCP-PMT optically coupled to a $(1.7 \mathrm{~cm})^{3}$ LYSO crystal recorded during an $8 \mathrm{GeV}$ electron run. 


\section{Timing in LYSO-based Calorimeters}

The timing measurement in LYSO-based calorimeters is driven by three main factors, other than the intrinsic transit time of the photodetector itself and the DAQ electronics: a) the shower profile fluctuations, b) the scintillation time, and c) the light propagation time. Stochastic processes during the development of an electromagnetic shower affect the time of the observed signals, as both the transverse size and the depth of the shower can fluctuate on an event-by-event basis. Random processes in the scintillation mechanism and the randomization of the optical paths for the scintillation light affect both the speed of the signal formation and the time jitter. We studied these effects using two independent experimental setups.

For a homogeneous crystal calorimeter, we were interested in the characterization and optimization of the light propagation time, i.e., the time that the scintillation light spends to travel down the whole length of the crystal. Our setup used a small LYSO cube with linear dimensions of $17 \mathrm{~mm}$ as the active scintillation element. The size of this element reduced the effect of the light propagation time and jitter. The LYSO cube was placed behind approximately $4.5 X_{0}$ radiation lengths of lead. Using this LYSO-based sampling calorimeter, we measured the time resolution of the electrons.

We also investigated a shashlik calorimeter composed of alternating layers of tungsten and LYSO, in which the scintillation light was extracted through wavelength shifting (WLS) fibers. In this setup, the light propagation time through the fiber is the dominant factor of the timing measurement. We studied, as a baseline, an alternate version of this calorimeter, in which the light was extracted through a direct optical coupling of the photodetectors at the edges of a few LYSO layers to minimize the light propagation time.

\subsection{Timing Studies of the LYSO-based Sam- pling Calorimeter}

We studied the combined impact of the shower profile fluctuations, scintillation mechanism in LYSO, and light propagation time resolution using a sampling calorimeter with a $(1.7 \mathrm{~cm})^{3}$ LYSO cube as the active element. The LYSO crystal was wrapped in Tyvek, and attached to the Hamamatsu R3809 MCP-PMT (HAMB) with optical coupling [13]. A second Hamamatsu MCP-PMT photodetector 


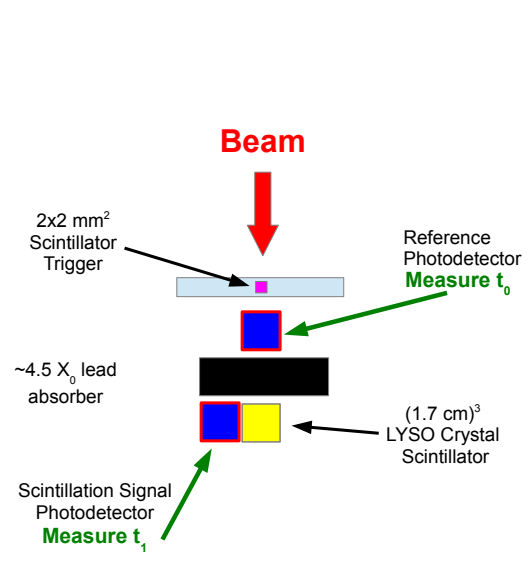

(HAMA) was placed upstream of the calorimeter and used to measure the reference time. A schematic diagram and a photograph of the experimental setup are shown in Figure 5.

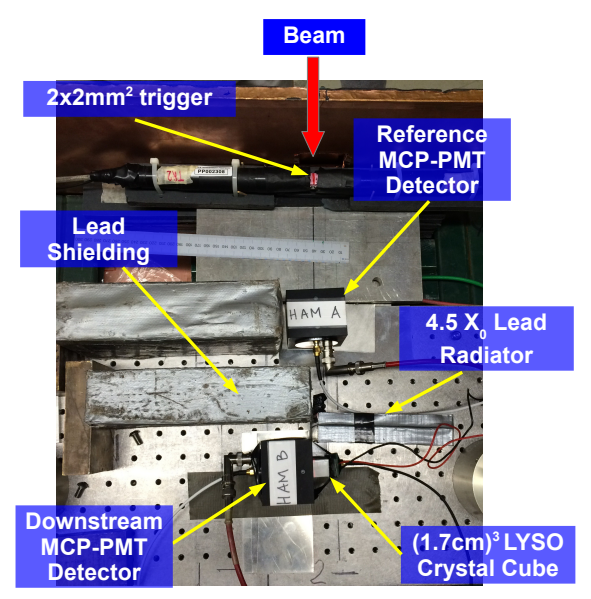

Figure 5: Schematic diagram of the experimental setup for the time-of-flight measurement using the LYSO sampling calorimeter (left), along with a picture of the experimental setup (right).

To ensure that the electron beam was constrained to within a $2 \times 2 \mathrm{~mm}^{2}$ region, a plastic scintillator placed upstream and approximately $2 \mathrm{~mm}$ by $2 \mathrm{~mm}$ in cross-sectional area was used to trigger the DAQ readout on the DRS digitizer. The electron events were identified by requiring a signal with amplitude larger than $10 \mathrm{mV}$ in a Cherenkov counter located upstream. Large lead bricks were placed upstream of the Hamamatsu R3809 MCP-PMT (HAMB), out of the path of the beam. These shielded the photodetector from stray particles produced in events where an electromagnetic shower occurred upstream of the lead radiator. Such stray shower particles yielded very fast signals that could significantly contaminate the scintillation signal. Using the same experimental setup without the LYSO active element in place, we found that the stray shower type events yielded less than $10 \%$ contamination, causing a negligible effect on the scintillation signal.

The thickness of the LYSO active element was relatively small and captured only a fraction of the total energy of the electron, but yielded a reasonable energy measurement, as it is close to the shower maximum. 
The time-of-flight measurement was performed using the LYSO sampling calorimeter for electron beams with energies varying from $4 \mathrm{GeV}$ to $32 \mathrm{GeV}$. The corresponding measured time-of-flight distributions are shown in Figure 6. We achieved the best time resolution of $34 \mathrm{ps}$ for electrons with beam energy of $32 \mathrm{GeV}$.
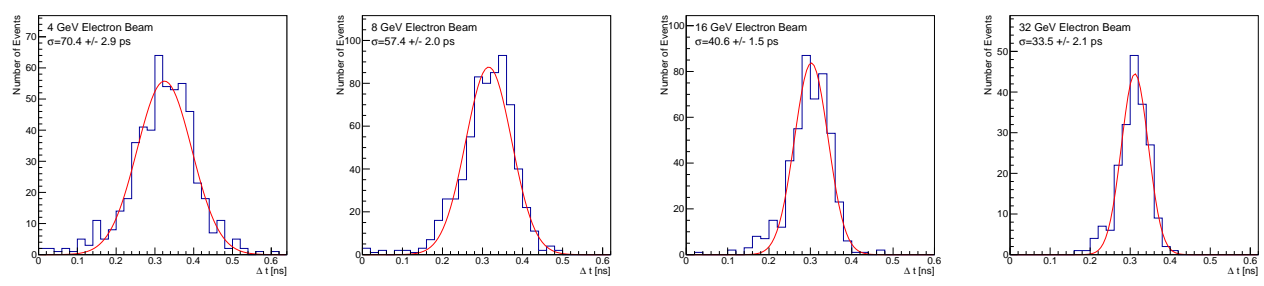

Figure 6: Time-of-flight distributions for the LYSO cube sampling calorimeter for $4 \mathrm{GeV}$ (top left), $8 \mathrm{GeV}$ (top right), $16 \mathrm{GeV}$ (bottom left), $32 \mathrm{GeV}$ (bottom right) electron beam energies.

The time resolution measurement is plotted as a function of the beam energy in Figure 15 (left). We fitted the result to the sum of a $1 / \sqrt{E}$ term and a constant term of about 11 ps. Given that we measured the contribution to the intrinsic time resolution of the photodetector and the DAQ electronics to be about 20 ps [9], using the results from the $32 \mathrm{GeV}$ electron beam, we infer that the combined contribution to the time resolution from the shower profile fluctuations, the scintillation mechanism, and the light propagation time inside the LYSO cube is about 27 ps.

\subsection{Timing Studies of the LYSO-Tungsten Shash- lik Calorimeter}

\subsubsection{Wavelength shifting fibers readout (WLS Y11 \& DSB1)}

We studied the time resolution of a LYSO-tungsten shashlik calorimeter, which is one of the proposed choices for the Phase 2 upgrade of the CMS endcap calorimeter system [7]. We compared the time resolution performance for two alternative light propagation schemes.

In our setup the scintillation light was collected by WLS fibers that passed through a set of four holes in the LYSO and tungsten layers. 
In Figure 7, a shashlik cell and the light extraction scheme are illustrated. A schematic diagram and a photograph showing this experimental setup are shown in Figure 8. Two MCP-PMTs by Hamamatsu (R3809) were used to collect the scintillation light, while a Photek 240 MCP-PMT was used as a reference time detector.

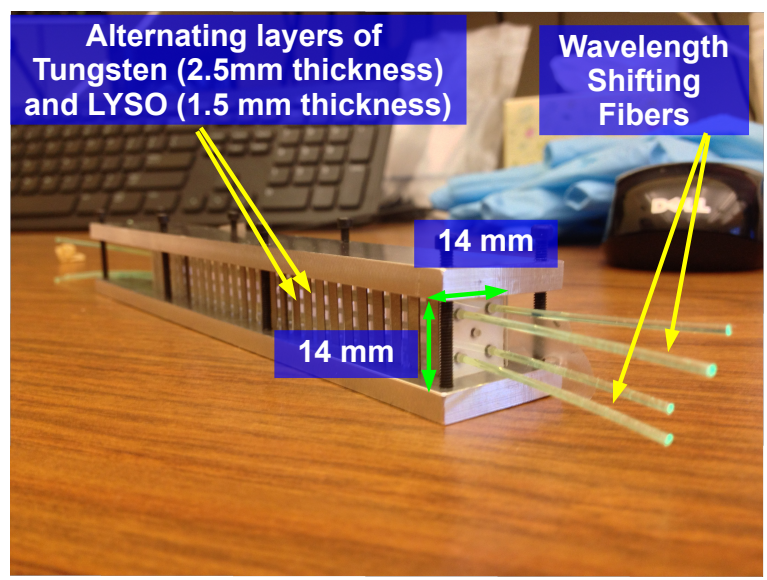

Figure 7: The shashlik configuration based upon interleaved W and LYSO layers. Twenty-eight LYSO crystal plates and twenty-seven $\mathrm{W}$ plates comprise the module. Four WLS fibers are used to read out the scintillation light from the tiles. 

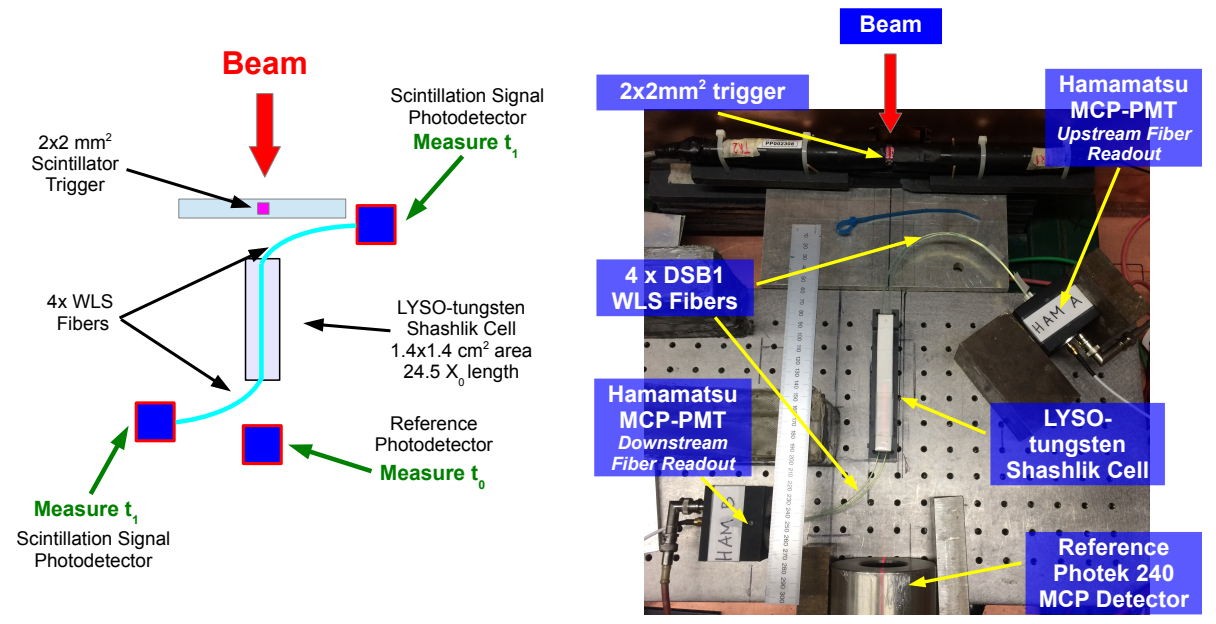

Figure 8: Schematic diagram of the experimental setup for the time-of-flight measurement using the LYSO-tungsten shashlik calorimeter with fiber signal extraction (left), along with a photograph of the experimental setup (right).

We compared the signal pulses obtained using two different types of WLS fiber in the same LYSO-tungsten shashlik calorimeter. Figure 9 (a) and (b) shows the pulse shapes averaged over a few hundred events obtained using DSB1 fibers [14] and Y11 fibers, plotted in blue and red, respectively. We found that the rise time of the pulse obtained using the DSB1 fibers, approximately $2.4 \mathrm{~ns}$, is significantly faster than the rise time of the pulse obtained using the Y11 fibers, which is approximately $7.1 \mathrm{~ns}$. Thus, to optimize the time resolution of this type of calorimeter, the DSB1 fiber provides a better choice than Y11, if only this parameter is considered. The signal rise times we observed are comparable to the measured decay times of the corresponding WLS fibers [14. 

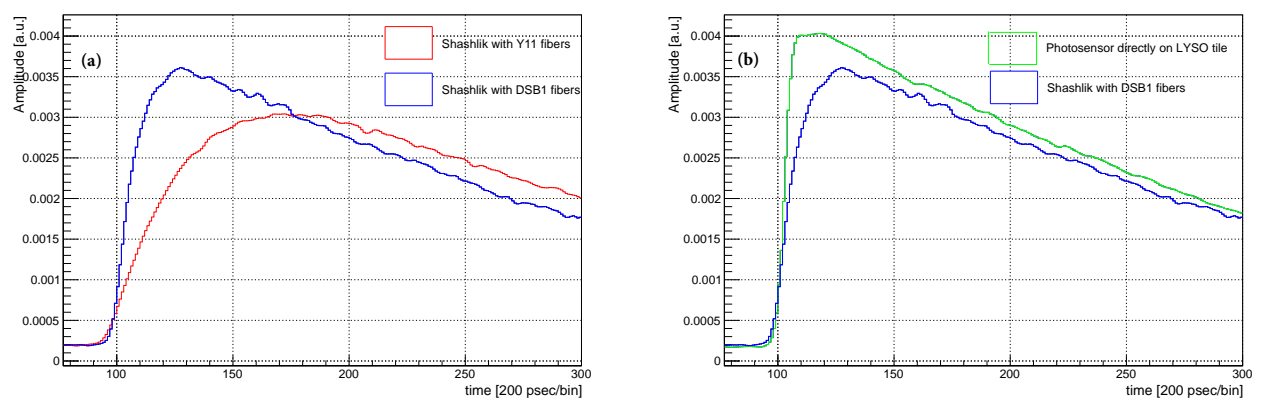

Figure 9: (a) Pulse shapes digitized by the DRS4 board and averaged over several hundred events obtained from the LYSO-tungsten shashlik calorimeter with light extracted using DSB1 (blue) and Y11 (red) WLS fibers. (b) DSB1(blue) shashlik average light pulse shape compared with the averaged pulse shape obtained from direct optical coupling of the photodetector to one edge of a LYSO tile in the shashlik calorimeter (green).

Using the shashlik calorimeter cell with DSB1 fibers, we measured the time resolution for electron beams with energy varying between $4 \mathrm{GeV}$ and $32 \mathrm{GeV}$. Figure 10 (b) shows the distribution of the pulse integral, which is proportional to the total collected charge, for the $32 \mathrm{GeV}$ beam; an energy resolution of approximately $5 \%$ was observed, whereas for the small LYSO cube, shown in 10 (a), the energy resolution was about 20\%. For this particular run in the Shashlik setup, no electron identification requirements could be made because of a misconfiguration of the upstream Cherenkov counter; therefore, the background is visible.

The time-of-flight distributions, fitted to Gaussian functions, are shown in Figure 11, and the $\sigma$ parameter of the Gaussian fit is plotted as a function of the beam energy in Figure 15. We found that the dependence of the time resolution on the beam energy follows a $1 / \sqrt{E}$ functional form, indicating that the current calorimeter setup remains in the photostatistics-limited regime. The best time resolution we obtained with this setup is 104 ps. As the measurements are photostatistics limited, the result may be improved in the future if the light collection efficiency will be increased. 

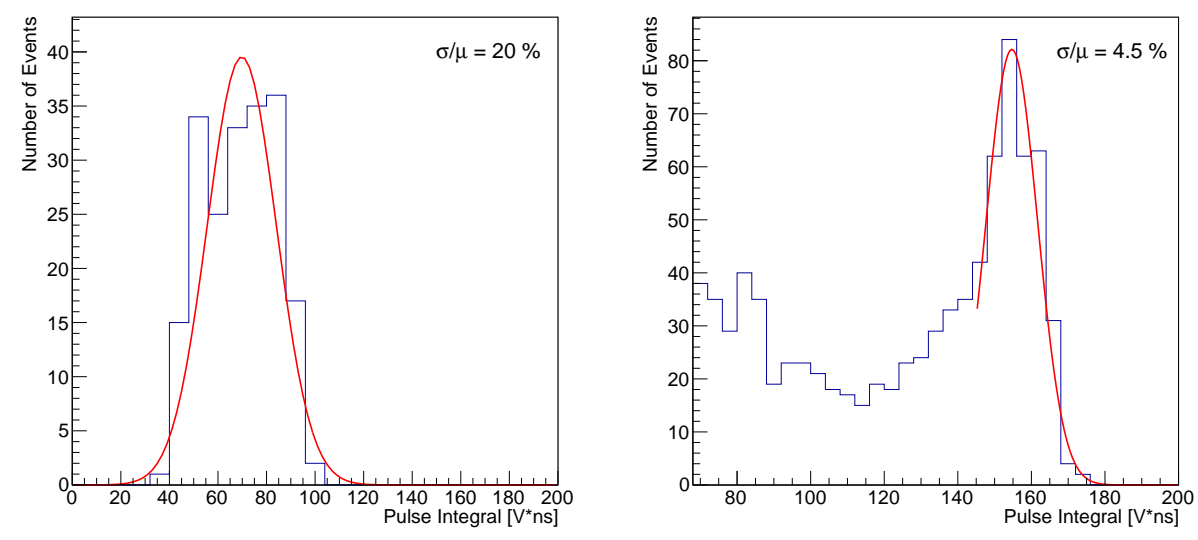

Figure 10: (Left) Histogram of the pulse integral, which is proportional to the total collected charge, for events recorded using the LYSO cube sampling calorimeter for a $32 \mathrm{GeV}$ electron beam. (Right) Histogram of the pulse integral for events recorded using the LYSO-tungsten shashlik calorimeter using DSB1 fibers for a $32 \mathrm{GeV}$ electron beam. The background is included because of a misconfiguration of the Cherenkov counter.
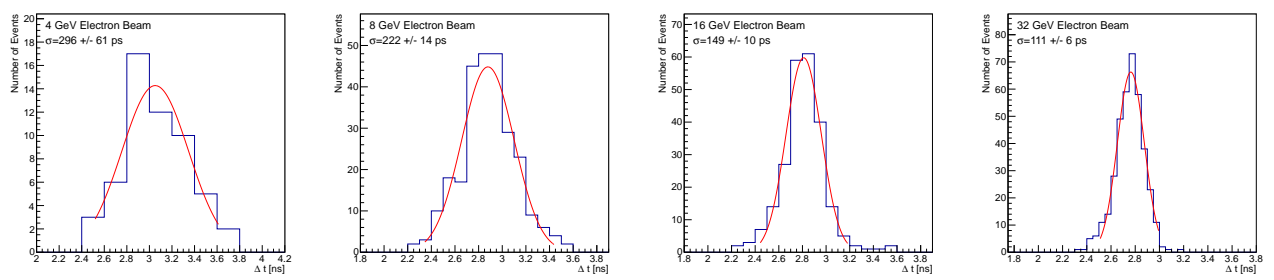

Figure 11: Time-of-flight distributions for the LYSO-tungsten shashlik calorimeter using DSB1 fibers for electron beams with varying beam energies.

\subsubsection{Directly coupled MCP-PMTs to LYSO shashlik plates}

In this setup, the MCP-PMT photodetectors were directly coupled to the edges of two adjacent LYSO layers in the shashlik calorimeter, and the scintillation light was directly transported to the photodetector through the edges of the tile layers. A schematic diagram and corresponding picture of the experimental setup are shown in Figure 12 
Figure 13 shows a zoomed-in photograph of the exposed LYSO plates from which the scintillation light signal was extracted.
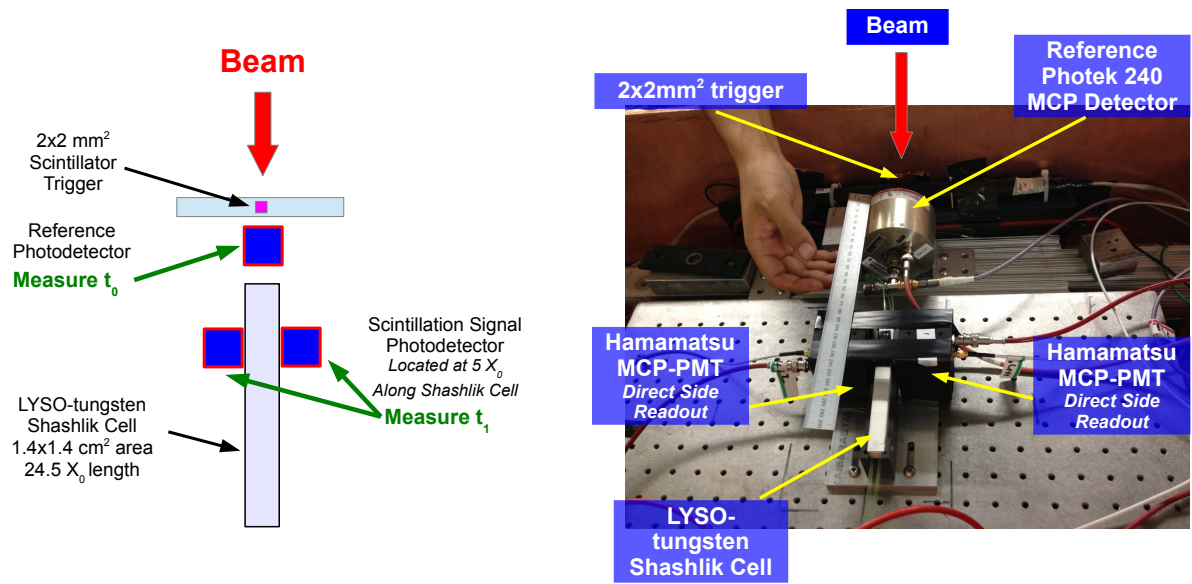

Figure 12: Schematic diagram of the experimental setup for the time-of-flight measurement using the LYSO-tungsten shashlik calorimeter with signal extraction from the edges of two LYSO plates (left), along with a picture of the experimental setup (right).

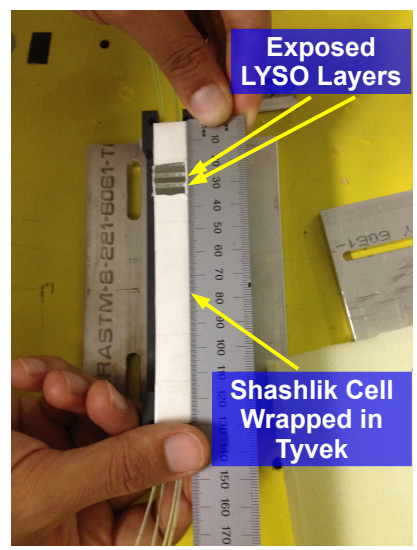

Figure 13: Photograph of the two exposed LYSO layers in the shashlik cell. The scintillation light signal is extracted by optically coupling the edges of these two exposed LYSO layers to MCP-PMT photodetectors.

With this setup, we invoke an interplay between the light propagation jitter and the limited photostatistics. By placing the photodetec- 
tors in direct contact with the edges of two LYSO layers, we minimized the distance the scintillation light travels to reach the photodetectors, and reduced the impact of the light propagation jitter on the time measurement resolution. However, in this setup, we also reduced the available photostatistics, as we collected the light from only a small fraction of the shashlik cell. Figure 14 shows the time-of-flight distributions for electron beams at various energies, fitted to Gaussian functions. The width of the best-fit Gaussian is plotted as a function of the beam energy in Figure 15. The best time resolution that we obtained is about 55 ps; fitting the result to the sum of a $1 / \sqrt{E}$ term and a constant term, we found a constant term of about $30 \mathrm{ps}$.
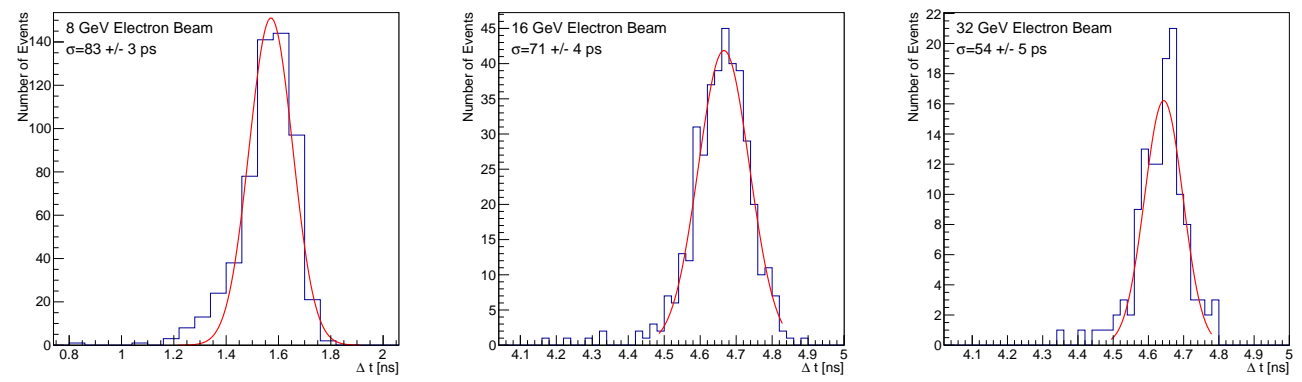

Figure 14: Time-of-flight distributions for the LYSO-tungsten shashlik calorimeter with signal extracted from the edges of two LYSO layers. 

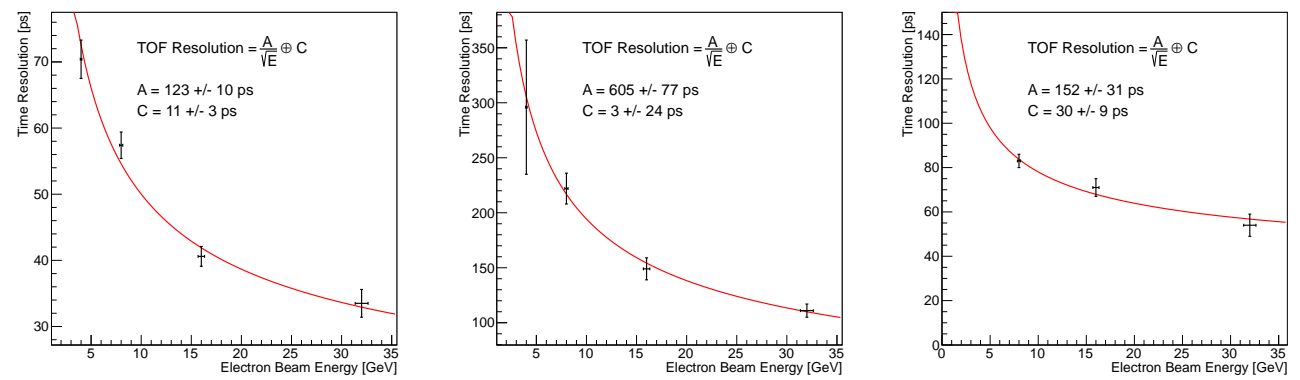

Figure 15: Timing resolution measurement as a function of the electron beam energy for (left) the LYSO cube sampling calorimeter, (middle) the LYSO-tungsten shashlik calorimeter read-out with DSB1 fibers, (right) the LYSO-tungsten shashlik calorimeter read-out directly by optically coupling to the edges of two LYSO layers. In all cases, we fit the data with a function of $1 / \sqrt{E}$ and a constant term.

In summary, we found that removing the impact of the wavelength shifting mechanism and minimizing the impact of optical transit do indeed improve the time resolution, but at a cost in photostatistics. The results obtained in this experiment suggest that a LYSO-tungsten shashlik calorimeter with edge readout can likely achieve a 30 ps resolution provided some improvement to the light collection efficiency is achieved.

\section{Results Discussion and Summary}

In this article, we have analyzed the results of a set of studies characterizing the timing performance of LYSO-based calorimeters. Using a $(1.7 \mathrm{~cm})^{3}$ LYSO crystal that samples the electromagnetic showers created by electrons of various energies ranging from $4 \mathrm{GeV}$ to $32 \mathrm{GeV}$ at about $4.5 X_{0}$, we infer that the contribution to the time resolution from event-by-event fluctuations of the shower profile, scintillation process, and light propagation is less than $30 \mathrm{ps}$. Studies using different WLS fibers in a LYSO-tungsten shashlik calorimeter demonstrate that the choice of the fiber affects the timing performance. Besides the absorption and re-emission processes in the fibers, we found that another important factor influencing the timing performance is the light extraction efficiency. Using DSB1 fibers, despite being photo- 
statistics limited, the best time resolution obtained was equal to approximately $100 \mathrm{ps}$. A future development of this detector will be focused on increasing the light collection efficiency. In a setup where the scintillation light from the LYSO-tungsten shashlik calorimeter is extracted via the edges of two LYSO layers, thereby removing completely the WLS mechanism and long light propagation distance, the best time resolution achieved was $55 \mathrm{ps}$. This result indicates that this calorimeter design can achieve the 30 ps time resolution benchmark obtained with the LYSO cube, provided some improvement to the light collection efficiency is achieved[A9].

In comparing results using different light extraction schemes, we found that, at a given light yield, the time resolution depends significantly on the light propagation fluctuations. As the light yield increases, the dependence on the light propagation fluctuations is reduced. The effect can be seen in the summary Figure 16, which shows the dependence of the time resolution on the average pulse height for the shashlik cell with light extracted through the DSB1 fibers, and for the sampling calorimeter with the LYSO cube. For the same average pulse height of $500 \mathrm{mV}$, the LYSO cube time resolution is about half of the time resolution of the shashlik using the DSB1 fibers, which have also twice the rise time. As the pulse height increases, the time resolution improves. Extrapolating to the regime of very large light yields, we should be able to reach asymptotically the best resolution without limitations from the light propagation fluctuations. 


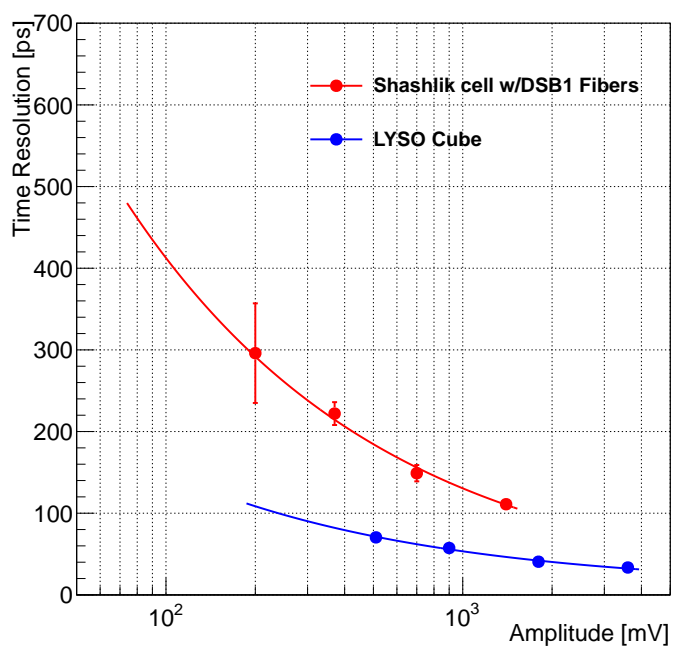

Figure 16: Comparison of the time resolutions obtained with the $(1.7 \mathrm{~cm})^{3}$ LYSO cube (blue), and the LYSO-tungsten shashlik calorimeter with light extracted using DSB1 fibers (red). The x-axis displays the amplitude of the signal, corrected for the attenuation factors.

In summary, using a LYSO-based calorimeter and different light propagation experimental setups, we obtained an approximately $30 \mathrm{ps}$ resolution time measurement for the maximum light yield achieved. As a follow-up, we will investigate the time resolution in the limit of a very large light yield, and attempt to improve the light collection efficiency in these types of detectors.

\section{Acknowledgements}

We would like to thank Erik Ramberg and Sergey Los for their help and support of this work, and Aria Soha and the FTBF test beam facility for the beam delivery and control. We thank Randy Ruchti for providing us with the DSB1 fibers used in the measurements, and Eileen Hahn for the high quality work in polishing the fibers. We would also like to thank Ewa Skup and Geoff Savage for helping with the operation of the Cherenkov counters, and Todd Nobel for organizing and providing the supporting equipment at FTBF.

This work is supported by funding from Fermi Research Alliance, LLC under Contract No. DE-AC02-07CH11359 with the United States 
Department of Energy and from California Institute of Technology High Energy Physics under Contract DE-SC0011925 with the United States Department of Energy.

\section{References}

[1] L. Rossi, and O. Brüning, "High Luminosity Large Hadron Collider A description for the European Strategy Preparatory Group," Tech. Rep. CERN-ATS-2012-236, CERN, Geneva, Aug 2012.

[2] L. Zhang, R. Mao, F. Yang, and R. Zhu, "LSO/LYSO Crystals for Calorimeters in Future HEP Experiments," IEEE Transactions on Nuclear Science, vol. 61, pp. 483-488, Feb 2014.

[3] R. Mao, L. Zhang, and R. Zhu, "Gamma ray induced radiation damage in PWO and LSO/LYSO crystals," in Nuclear Science Symposium Conference Record (NSS/MIC), 2009 IEEE, pp. 2045-2049, Oct 2009.

[4] J. Chen, R. Mao, L. Zhang, and R. Zhu, "Gamma-Ray Induced Radiation Damage in Large Size LSO and LYSO Crystal Samples," IEEE Transactions on Nuclear Science, vol. 54, pp. 1319 1326, Aug 2007.

[5] L. Zhang, R. Mao, and R. Zhu, "Effects of neutron irradiations in various crystal samples of large size for future crystal calorimeter," in Nuclear Science Symposium Conference Record (NSS/MIC), 2009 IEEE, pp. 2041-2044, Oct 2009.

[6] G. Dissertori, D. Luckey, Nessi-Tedaldi, et al., "Results on damage induced by high-energy protons in LYSO calorimeter crystals," NIM A 745 (2014) 1-6.

[7] D. Contardo and J. Spalding, "CMS Phase 2 Upgrade: Preliminary Plan and Cost Estimate," Tech. Rep. CERN-RRB-2013-124, CERN, Geneva, Oct 2013.

[8] W. W. Moses and S. E. Derenzo, "Prospects for Time-of-Flight PET using LSO Scintillator," IEEE Transactions on Nuclear Science, vol. 46, pp. 474-478, June 1999. 
[9] A. Ronzhin, S. Los, E. Ramberg, et al., "Development of a new fast shower maximum detector based on microchannel plates photomultipliers (MCP-PMT) as an active element," NIM A 759 (2014) 65-73.

[10] http://www.hamamatsu.com/resources/pdf/etd/R3809U-50_ TPMH1067E09.pdf.

[11] http://www.photek.com/pdf/datasheets/detectors/DS006_ Photomultipliers.pdf.

[12] S. Ritt, R. Dinapoli, and U. Hartmann, "Application of the DRS chip for fast waveform digitizing," NIM A 623 (2010) 486-488.

[13] http://www.ellsworth.com/dow-corning-q2-3067-optical-couplant-453g-bottle.

[14] M. Albrecht, K. Andert, P. Anselmino, et al., "Scintillators and Wavelength Shifters for the Detection of Ionizing Radiation," Proceedings of the 8th Conference on astroparticle, particle and space physics, detectors and medical physics applications, pp. 502-511, 2003. 